\title{
Ordered alloying of Pd with the Mo(100) Surface
}

\author{
D. Wu ${ }^{1,2}$ W. K. Lau, ${ }^{1}$ Z. Q. He, ${ }^{1}$ Y. J. Feng, ${ }^{1}$ M. S. Altman,,${ }^{1, *}$ and C. T. Chan ${ }^{1}$ \\ ${ }^{1}$ Department of Physics, Hong Kong University of Science and Technology, Clear Water Bay, Kowloon, Hong Kong \\ ${ }^{2}$ Department of Physics, Fudan University, Shanghai, China
}

(Received 26 April 2000)

\begin{abstract}
The interaction of Pd with the Mo(100) surface has been studied with low-energy electron microscopy and diffraction, and first-principles total-energy calculations. Deposition of Pd at elevated temperature leads initially to the development of an intense $c(2 \times 2)$ diffraction pattern. Theoretical calculations demonstrate that a $c(2 \times 2)$ substitutional alloy is energetically favored compared to an overlayer structure at half monolayer coverage. The creation of a large number of islands upon Pd deposition is consistent with the formation of a substitutional alloy. Accommodation of Pd in excess of the ideal $c(2 \times 2)$ coverage leads to the formation of a $c(2 \times n)$ structure $(n=8,10)$, although with little apparent further change of surface morphology. Proliferation of antiphase domain walls in the $c(2 \times 2)$ alloy is proposed to explain the $c(2 \times n)$ periodic structure. Theoretical calculations indicate that a pseudomorphic Pd overlayer is the most stable configuration at 1 monolayer coverage, and that the Pd-covered $\mathrm{Mo}(100)$ surface may become unstable with respect to faceting to the $\{112\}$ orientations if pseudomorphic growth can be realized up to 2 monolayer coverage. However, the $c(2 \times n)$ structure is found experimentally to be stable at the interface between Mo and thicker Pd overlayers. These results suggest that there is a kinetic limitation to the formation of the pseudomorphic structure and the faceting instability is preempted by the formation of more complex surface alloy structures.
\end{abstract}

\section{INTRODUCTION}

Local free-energy minima for the low-index (110), (211), (100), and (111) planes of bare $\mathrm{W}$ and Mo are responsible for their thermodynamic stability and appearance on the equilibrium crystal shape. ${ }^{1}$ These surfaces have differing degrees of atomic roughness which are reflected to some extent in their relative work functions $\Phi_{110}>\Phi_{211}>\Phi_{100}>\Phi_{111} \cdot{ }^{2}$ The stability of these surfaces with respect to metal overlayer growth shows similar trends. Since the surfaces of W and Mo have high surface energy, it is fairly easy to grow films of materials having lower surface energy on top of them in pseudomorphic or other ordered structures. ${ }^{3}$ The largest variety of periodic overlayer structures forms on the (110) surface, presumably because it is atomically the smoothest. ${ }^{3,4}$ On the other hand, the (111) surface is atomically the roughest and notably less stable than the (110). Although ordered overlayers form at low temperature, the (111) surface is quite prone to faceting in the presence of numerous metal species 5 including Pd (Refs. 6 and 7) at high temperature. Faceting occurs predominantly with $\{211\}$ character, although $\{110\}$ faceted structures have also been observed to a lesser extent. Both types of facets have been accounted for by theory. ${ }^{7-9}$

Intermediate between the (110) and (111) lie the (211) and (100) surfaces. While there have been no reports of metal-induced faceting in the macroscopic scale, many ordered metal overlayer structures have been observed on these surfaces. ${ }^{3,10}$ Particularly unique among them is a $c(2 \times 2)$ structure that forms on the $\mathrm{W}(100)$ surface upon annealing 0.5 monolayer (ML) of $\mathrm{Cu}, \mathrm{Au}, \mathrm{Ag}$, or Pt in the range 500$700 \mathrm{~K}$. Although originally believed to be an overlayer structure, ${ }^{11}$ this $c(2 \times 2)$ has more recently been shown by experiment to be a two-dimensional substitutional surface alloy [Fig. 1(a)]. ${ }^{12,13}$ In contrast, the intense $c(2 \times 2)$ diffraction patterns that form as the result of annealing 0.5 ML of $\mathrm{Cu}$ and $\mathrm{Ag}$ on $\mathrm{Mo}(100)$ (Ref. 14) were interpreted in a low- energy electron diffraction (LEED) intensity analysis in terms of overlayer structures. ${ }^{15}$ The possibility of a surface alloy was not considered. This is somewhat surprising given the extensive experimental evidence of $c(2 \times 2)$ alloys induced by noble metals on $\mathrm{W}(100)$, with the exception of Pd. ${ }^{16-18}$ For Pd on W(100), only very weak $\frac{1}{2}$-order diffraction features characteristic of a $c(2 \times 2)$ structure were ever observed in LEED. ${ }^{19}$ However, it is not entirely clear whether the annealing or deposition temperatures that were employed in that work were appropriate for the formation of a well-ordered $c(2 \times 2)$ alloy. On $\mathrm{Mo}(100)$, annealing of 0.5 ML of Pd to $600 \mathrm{~K}$ caused only a "fuzzy" $c(2 \times 2)$ LEED pattern. ${ }^{20}$ This result was interpreted in terms of randomly distributed $c(2 \times 2)$ overlayer islands rather than the surface alloy structure.

The present work is aimed at revisiting $\mathrm{Pd} / \mathrm{Mo}(100)$ in order to search more systematically for the $c(2 \times 2)$ structure at $0.5 \mathrm{ML}$ coverage and to distinguish the nature of this structure, whether two-dimensional substitutional alloy or overlayer. Using LEED, growth conditions are found in this work that lead to well-ordered $c(2 \times 2)$ and more complex $c(2 \times n)$ structures. First-principles total-energy calculations clearly demonstrate that the $c(2 \times 2)$ is a surface alloy. Proliferation of antiphase domain walls in the $c(2 \times 2)$ alloy is proposed to explain the new $c(2 \times n)$ periodic structure. Low-energy electron microscopy (LEEM) observations of surface morphological changes that accompany the formation of these structures provide additional experimental evidence of surface alloys. We also explore experimentally and theoretically the possibility for somewhat more drastic Pdinduced modification of $\mathrm{Mo}(100)$ via faceting, analogous to metal-induced faceting of Mo and W(111) surfaces.

\section{THEORY}

The Pd on Mo(100) calculations were performed within the framework of local-density-functional formalism. ${ }^{21}$ 
(a)
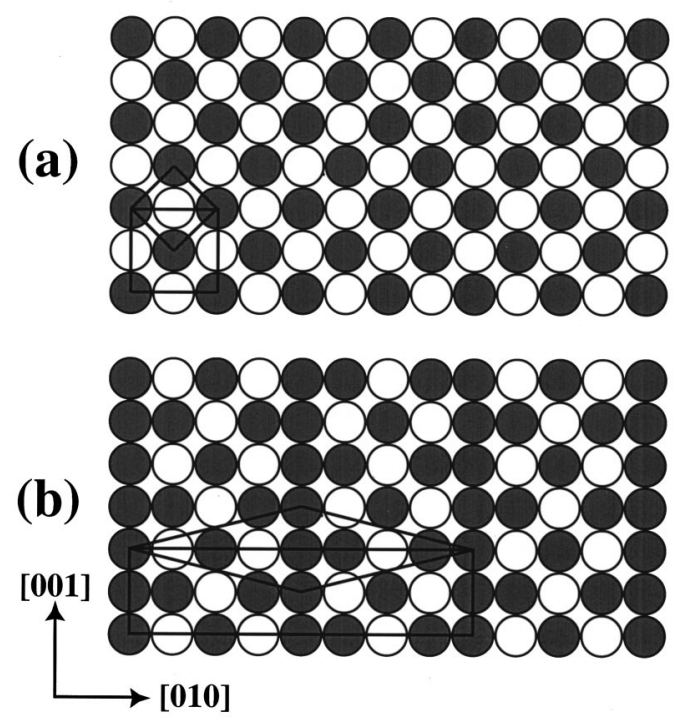

FIG. 1. Pd (shaded) and Mo (unshaded) atoms in the topmost layer of the (a) $c(2 \times 2)$ and (b) $c(2 \times 8)$ substitutional alloys at the $\mathrm{Mo}(100)$ surface.

Norm-conserving pseudopotentials were used. ${ }^{22}$ The basis consisted of plane waves with kinetic energy up to a cutoff of $11.5 \mathrm{Ry}$ together with Bloch sums of numerical orbitals centered at atomic sites. ${ }^{23}$ We used standard repeated slab geometry, with a vacuum of $9.2 \AA$. Most of the calculations were done with slabs (substrate plus adlayers) that were nine layers thick. Thicker slabs (11 layer substrate plus adlayers) were used when the faceting instability was considered. These details will be specified also below. Pseudomorphic $\mathrm{Pd}$ adlayers or Mo/Pd alloy layers were added on both sides of the Mo substrate. We used a $c(2 \times 2)$ unit cell for all the calculations. $(4 \times 4)$ and $(12 \times 12)$ grids in the twodimensional (2D) Brillouin zone were used for $k$-point sampling and will be specified in the following results. All atomic coordinates were relaxed by computing the Hellmann-Feynman forces. A Broyden algorithm, together with a convergence-acceleration scheme ${ }^{24}$ was used to find the equilibrium atomic coordinates during the atomic relaxation procedures.

We first consider the energetics of the interaction of $\mathrm{Pd}$ with $\mathrm{Mo}(100)$. The results are summarized in Fig. 2. For a given configuration of Pd on the Mo substrate, we define a surface formation energy per substrate surface atom $\left(E_{s}\right)$ as

$$
E_{s}=\frac{1}{2 N}\left[E_{(\mathrm{Pd} / \mathrm{Mo}) \text { slab }}-\left(E_{(\mathrm{Mo}) \text { substrate }}+n \mu_{\mathrm{pd}}\right)\right]+\sigma,
$$

where $N$ is the number of surface atoms per surface unit cell, $E_{(\mathrm{Pd} / \mathrm{Mo}) \text { slab }}$ is the total energy per unit cell of the slab with Pd adsorbed on Mo, $E_{(\text {Mo)substrate }}$ is the total energy of the clean Mo substrate, $n$ is the total number of Pd atoms in the surface unit cell, $\mu_{\mathrm{Pd}}$ is the chemical potential of the Pd atoms, and $\sigma$ is the surface energy per surface atom of the clean Mo substrate. The factor of 2 in the denominator accounts for the fact that we put Pd atoms on both sides of the Mo slab.

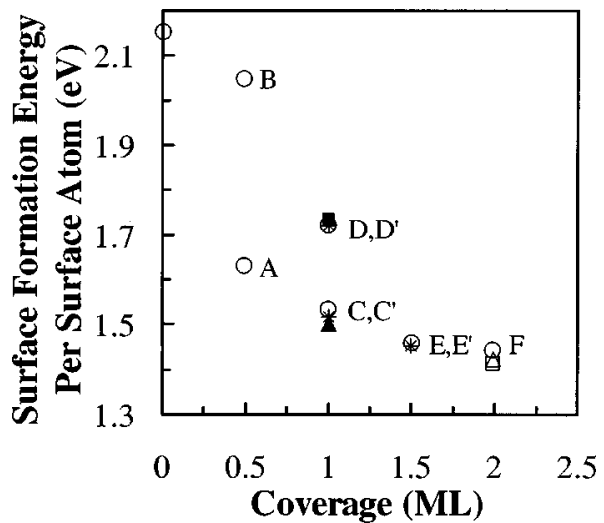

FIG. 2. Surface formation energies of $\mathrm{Pd}$ on $\mathrm{Mo}(100)$ for various coverages and configurations. See text for the explanation of the atomic arrangement in different configurations. The circles are for $\mathrm{Pd} / \mathrm{Mo}(100)$ calculated with a sparse $(4 \times 4) k$-point grid, while the asterisks are the same configurations calculated with a (12 $\times 12$ ) grid. At 1 ML Pd coverage, the solid triangle and solid square are for one pseudomorphic layer of $\mathrm{Pd}$ on $\mathrm{Mo}(100)$ and $\mathrm{Mo}(211)$, respectively. At $2 \mathrm{ML} \mathrm{Pd}$ coverage, the open triangle and open square are for two pseudomorphic layers of $\mathrm{Pd}$ on $\mathrm{Mo}(100)$ and $\operatorname{Mo}(211)$, respectively.

For the same Pd coverage, the surface formation energy for different Pd configurations (e.g., overlayer, alloy, or subsurface) can be compared directly and whichever configuration has lower energy is the energetically favorable one. Comparing results at different coverages requires the knowledge of $\mu_{\mathrm{Pd}}$. In Fig. 2, $\mu_{\mathrm{Pd}}$ is set equal to the bulk energy of $\mathrm{Pd}$ in the fcc structure. The choice of the bulk energy as the reference energy of the adatoms has the advantage that we know immediately whether the adatoms wet the substrate or not. If $E_{s}<\sigma$, then the Pd atoms gain energy by covering the surface rather than forming macroscopic bulklike islands on top of the substrate. In that case, we can conclude that the adatoms wet the substrate. In contrast, if the surface formation energy increases when covered with $\mathrm{Pd}$, it should be energetically more favorable for the adatoms to be in a bulk Pd island. In this way, we can deduce the number of thermodynamically stable adlayers that can wet the substrate pseudomorphically.

In Fig. 2, we compare $E_{s}$ for various $\mathrm{Pd} / \mathrm{Mo}$ configurations and coverages. The circle at zero coverage represents the surface energy of the clean $\mathrm{Mo}(100)$ surface. ${ }^{25}$ The other symbols represent various plausible configurations with $\mathrm{Pd}$ overlayer atoms covering or substituting the surface Mo atoms within a $c(2 \times 2)$ surface unit cell. The atomic arrangements and coverages in these configurations are as follows: A, 0.5 ML, Pd atoms substituting for Mo surface atoms in the top layer forming an ordered $c(2 \times 2)$ substitutional surface alloy [Fig. 1(a)]; $B, 0.5 \mathrm{ML}, \mathrm{Pd}$ in a $c(2 \times 2)$ array on the fourfold-hollow sites above the Mo surface; $C, 1.0 \mathrm{ML}$, pseudomorphic Pd layer on top of the first layer of Mo; $D$, 1.0 ML, Pd in $c(2 \times 2)$ array on fourfold sites above a $c(2$ $\times 2$ ) ordered substitutional surface alloy in the top layer, i.e., an extra half monolayer of Pd over configuration $A ; E, 1.5$ ML, a full pseudomorphic Pd layer above a $c(2 \times 2)$ ordered substitutional surface alloy in the top layer, i.e., a full pseudomorphic Pd layer on top of configuration $A$; and $F$, 
2.0 ML, two pseudomorphic layers of Pd covering the Mo substrate. All the open circles represent $E_{s}$ for configurations $A-F$ calculated with a sparse $(4 \times 4)$ Monkhorst-Pack $k$-point grid. Configurations $C^{\prime}, D^{\prime}$, and $E^{\prime}$ that are marked by asterisks in Fig. 2 have the same atomic configurations as $C$, $D$, and $E$, respectively, but are calculated with a finer $(12 \times 12) k$-point grid. The energy differences between the coarse and the fine grid results are much smaller than the energy differences between different atomic configurations, so that the $k$-point sampling used in the calculation is adequate in the energy scale we are interested in. We have also considered several other configurations in which the Pd atoms penetrate and substitute Mo atoms in deeper layers. We found that whenever Pd atoms substitute Mo atoms in deeper layers (anywhere other than the top layer), the energy becomes so unfavorable that there is no point to relax the atomic configurations to their full equilibrium. These configurations are thus not shown in the figure. This is not surprising since fcc metals generally do not like to dissolve in refractory metals like $\mathrm{W}$ and Mo, which typically have much higher cohesive energy. The particularly low energy of a $c(2 \times 2)$ surface alloy, as exemplified by configurations $A$ and $E$, should be regarded as a peculiarity and will be explained below.

From Fig. 2, we can see that adding Pd atoms onto $\mathrm{Mo}(100)$ lowers its surface formation energy. Since the reference energy is the Pd bulk energy, this means that Pd wets $\mathrm{Mo}(100)$. This is consistent with the fact that Mo has a higher surface energy than Pd. At half-monolayer coverage, we note that a $c(2 \times 2)$ substitutional surface alloy model, configuration $A$, is substantially lower in energy than configuration $B$ in which half a layer of Pd atoms simply sits on top of the Mo surface. The extra stability of the $c(2 \times 2)$ top layer surface alloy is due to a rather subtle effect. It turns out that a $c(2 \times 2)$ array of surface vacancies on $\operatorname{Mo}(100)$ is comparable in energy ${ }^{26}$ to the $(1 \times 1)$ unreconstructed and Debe-King ${ }^{27}$ type $c(2 \times 2)$ reconstructed surfaces. Localdensity approximation (LDA) calculations show that electron depletion can further stabilize the surface vacancy array so that it becomes the ground state. ${ }^{26} \mathrm{We}$ note that $\mathrm{Pd}$ is more electronegative than Mo on the Pauling scale, and thus charge transfer from the Mo substrate to the Pd adatoms would stabilize the formation of a $c(2 \times 2)$ vacancy array on $\mathrm{Mo}(100)$. The Pd atoms would fill up the voids of the vacancies, leading to a smoother surface charge density profile that has lower electronic kinetic energy. There is certainly some metallic bonding between Pd and Mo in the alloy structure, but this bonding is not strong enough to disrupt the strong Mo-Mo bonding. The formation of the substitutional surface alloy owes a lot to the intrinsic stability of the vacancies on the Mo(100) surface and the surface-charging effect in the presence of the Pd adatoms. It is essentially a top-layer effect, and that is why there is no tendency for the alloy to form in the deeper layers. As a result, any configurations that involve Pd atoms substituting Mo atoms in deeper layers have very unfavorable energies.

For $1 \mathrm{ML}$ coverage, a complete pseudomorphic Pd layer covering the Mo substrate (configuration $C$ ) is more favorable than half a layer of Pd covering a Pd/Mo $c(2 \times 2)$ surface alloy (configuration $D$ ). This is quite understandable since in configuration $C$, the higher-surface-energy top-layer
Mo surface atoms are covered uniformly by Pd overlayer atoms that have lower surface energy. In addition, configuration $C$ has a smoother surface profile, leading to a lower kinetic energy of the electrons. It is interesting to see that the surface energy keeps lowering until the coverage becomes 2 ML as long as we consider pseudomorphic overlayers. In fact, examination of a ball-and-stick model of the bcc (100) surface reveals that the substrate atoms in the second layer are also exposed. Therefore, at a Pd coverage of $2 \mathrm{ML}$, the substrate Mo atoms are completely shielded from the vacuum. Thus, our results indicate that Pd should continue to wet the substrate surface up to at least $2 \mathrm{ML}$ as long as it is pseudomorphic. This is important in the context of faceting, which is discussed next.

We now turn to the question of the stability of the $\mathrm{Mo}(100)$ surface upon adsorption of Pd. It is well documented that while clean $\mathrm{W}$ and $\mathrm{Mo}(111)$ are stable, they become unstable with respect to faceting when covered by a physical monolayer [PML; all exposed substrate atoms in the topmost three "geometric layers" of the (111) surface are covered by adsorbate atoms] or equivalently three geometric ML's of some fcc metals such as Pd. ${ }^{6,7}$ The thermodynamic driving force can be traced to the increase in surface energy anisotropy when the surface is covered by an overlayer of fcc metals. ${ }^{8}$ That phenomena is not too surprising since the clean $\mathrm{Mo}(111)$ surface is just marginally stable with respect to faceting to $\{211\} .{ }^{9}$ The question we ask is whether the $\mathrm{Mo}(100)$ surface has the same type of instability. We first note that $\mathrm{Mo}(100)$ is stable when it is clean, and it appears on the Wulff plot of Mo. ${ }^{1,9}$ From previous local-density calculations ${ }^{8,9}$ we note that only two orientations can have a lower surface energy per unit cell than the (100) orientation for Mo, and they are $\{211\}$ and $\{110\}$. Since the (211) orientation makes a smaller angle with the (100) than does the (110), the increase of total surface area that would accompany faceting to (211) is less than the faceting to (110). Therefore we will consider $\{211\}$ here. $^{28}$

A bcc (100) surface would become thermodynamically unstable with respect to faceting to $\{211\}$ if the following condition is satisfied: ${ }^{29}$

$$
\frac{\gamma_{211}}{\cos \theta}<\gamma_{100}
$$

where $\gamma_{100}$ and $\gamma_{211}$ are the surface energies per unit area of the (100) and (211) orientations, respectively, and $\theta$ $=35.26^{\circ}$ is the angle between the two orientations. The $\cos \theta$ term on the left-hand side of Eq. (2) is a geometric factor that accounts for the increase of surface area upon faceting. When the Mo substrate is covered by Pd overlayers, the surface formation energies are understood as the heat of formation of the overlayer plus the surface energy of the bare substrate. If we choose to consider the surface energy per surface atom instead, Eq. (2) can be rewritten simply as

$$
E_{211}<E_{100},
$$

where $E_{100}$ and $E_{211}$ are the surface formation energies per surface atom as defined in Eq. (1). When a bcc (100) surface transforms to square-based pyramids (mandated by symme-

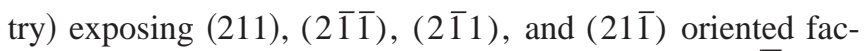
ets, the total surface area increases by a factor of $\sqrt{\frac{3}{2}}$. The 
(211) unit cell is precisely $\sqrt{\frac{3}{2}}$ times larger than the (100) unit cell. That means that the same number of Pd atoms that cover a (100) surface will also cover the $\{211\}$-faceted surface. This geometric fact underlies the simplicity of Eq. (3). This also means that it is meaningful to compare the surface formation energies for two different orientations directly, as the faceting transformation we are dealing with involves a constant number of Pd adatoms, and thus the results do not depend on the absolute value of the Pd chemical potential.

Since we are now comparing different orientations, we increase the slab and the vacuum thickness. The calculations involving faceting transformations are done with a vacuum thickness of $10.7 \AA$, and the slab contains an 11-layer thick Mo substrate, on the top and bottom of which we put the Pd overlayers. The $k$-point sampling is $(12 \times 12)$ in the twodimensional (2D) Brillouin zone. We compute the surface formation energies in the (100) and (211) directions and check to see if the condition $E_{211}<E_{100}$ is satisfied for a Pd coverage of $1 \mathrm{ML}$. The results are also shown in Fig. 2, where the solid triangle at $1 \mathrm{ML}$ Pd coverage represents $E_{100}$, the surface formation energy calculated for $\mathrm{Mo}(100)$ covered with one pseudomorphic layer of Pd. We note that the more accurate eleven-layer Mo substrate result (solid triangle) and the seven-layer Mo substrate result (configuration $C^{\prime}$, denoted by an asterisk in Fig. 2) are reasonably close together. The solid square represents $E_{211}$, the surface formation energy of $\mathrm{Mo}(211)$ covered with one pseudomorphic layer of Pd. We note that $E_{211}$ happens to be very close to the surface formation energy of configuration $D$ of the $M o(100)$ surface discussed previously, and that is a coincidence. What we see here is that the condition $E_{211}<E_{100}$ is clearly not satisfied, and therefore the $\operatorname{Mo}(100)$ surface is stable against faceting when it is covered by one pseudomorphic layer of $\mathrm{Pd}$. We found that although the surface formation energy $\gamma_{211}$ is actually lower than $\gamma_{100}$, the difference is not large enough to compensate for the $22.5 \%$ increase in surface area that would have to accompany a faceting transition from (100) to $\{211\}$. However, the situation changes when the Pd coverage is increased to $2 \mathrm{ML}$. The open triangle and open square in Fig. 2 represent $E_{100}$ and $E_{211}$, respectively, when the Mo substrate is covered by two pseudomorphic monolayers of Pd. We see that they have almost the same surface formation energy per surface atom. ${ }^{30}$ At 2 ML coverage, both Mo (100) and (211) substrate faces become completely shadowed by the overlayer Pd atoms. The increase in surface formation energy anisotropy is now big enough to compensate for the increase in surface area. We thus come to the conclusion that if $\mathrm{Pd}$ can be grown pseudomorphically and reaches a coverage of $2 \mathrm{ML}$ on $\mathrm{Mo}(100)$, then the substrate becomes potentially unstable with respect to faceting to $\{211\}$.

We need to remark here that the Herring condition for faceting [Eqs. (2) and (3)] are thermodynamic conditions, which means that if these conditions are satisfied, then the system gains energy upon faceting. However, it is known that the Herring condition exclusively considers the change in surface energy in the final state. Between the initial and final state, a large kinetic barrier associated with massive macroscopic transport of surface atoms must be overcome. In addition, there should be a nucleation barrier when the facets are small. ${ }^{8}$ Previous theoretical considerations showed that the Herring condition is just a necessary condition. In practice, faceting transformations are observed in metal-onmetal systems only when there is a fairly significant thermodynamic driving force, and are not observed for systems [such as $\mathrm{Ag} / \mathrm{Mo}(111)]$ that the Herring condition is marginally satisfied. ${ }^{8}$ In addition, the $35.26^{\circ}$ angle between a bcc (100) and (211) surface is large compared with the $19.47^{\circ}$ angle between a bcc (111) and (211) surface, where overlayer-induced faceting has been observed in many systems. With these considerations, it is not too likely that the faceting transformation can be realized in $\mathrm{Pd} / \mathrm{Mo}(100)$. Nevertheless, the potential for a faceting instability in the $\mathrm{Pd} /$ $\mathrm{Mo}(100)$ system is interesting and stimulates experimental investigation.

All the calculations are performed assuming pseudomorphic Pd layers. This is certainly an approximation that is mandated by the need of two-dimensional periodicity in our calculations. Such an approximation should be reasonable when we are considering the energetics of the adsorption of ultrathin overlayers in which every atom in the overlayer "sees" the substrate atoms and heat of formation is fairly high. However, if we were to consider the absorption of thicker layers, or if the heat of absorption is small, the overlayers can easily become incommensurate with the substrate. Density functional type calculations would then be impractical.

Lastly, we remark that the energetics of many fcc metals such as $\mathrm{Au}, \mathrm{Ag}$, and $\mathrm{Pt}$ interacting with Mo substrates are rather generic. The absolute energy scale and the minor details may change, but the corresponding surface formation energy diagrams are rather similar to Fig. $2 .{ }^{31}$ However, the stability of the surface against faceting is very adatom specific. $^{32}$

\section{EXPERIMENT}

The Mo sample was oriented to within $0.05^{\circ}$ from the (100) plane. It was cleaned by annealing to $1200 \mathrm{~K}$ in an oxygen pressure of $1 \times 10^{-7}$ torr and flashing to $2000 \mathrm{~K}$. Palladium was deposited from an electron-beam-heated rod. The homemade Pd source incorporated water cooling, shutter, and flux measurement by proportional ion current. The base pressure in the chamber was $1.4 \times 10^{-10}$ torr and the pressure rose to the mid- $10^{-10}$-torr range during deposition. The sample temperature was measured by a W/Re3\%-W/ Re $25 \%$ thermocouple attached to the side of the sample. The thermocouple temperature measurement was crossreferenced to infrared pyrometer $(700-1100 \mathrm{~K})$ and disappearing filament pyrometer $(1050-1250 \mathrm{~K})$ measurements. The experiments were carried out in a low-energy electron microscope. Contrast, resolution, and the operational principle of LEEM have been described previously. ${ }^{33,34}$ In summary, LEEM images surfaces with elastically backscattered, low-energy electrons. The lateral resolution of the instrument used in this work was $15 \mathrm{~nm}$. The capability to image monatomic surface steps gives LEEM atomic resolution perpendicular to the surface. ${ }^{34}$

\section{A. Lateral structure}

The LEED pattern of the clean Mo(100) surface at room temperature exhibited sharp $(1 \times 1)$ diffraction spots in ad- 

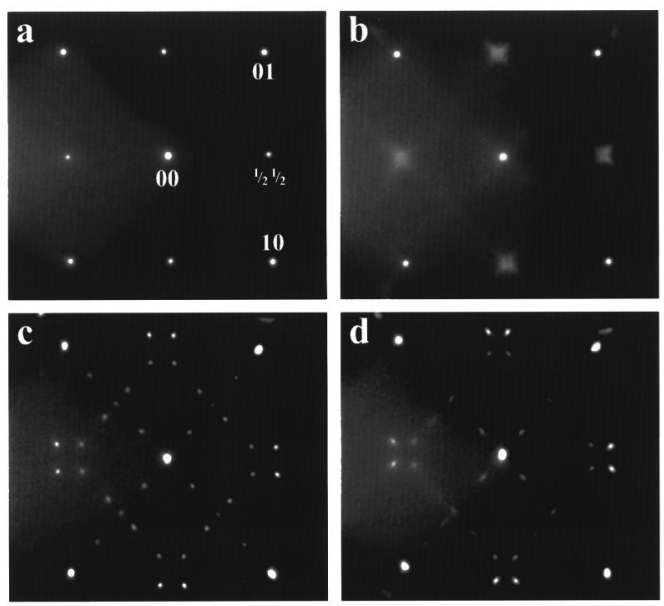

FIG. 3. LEED pattern of $\mathrm{Mo}(100)-\mathrm{Pd}$ structures (electron energy): (a) $c(2 \times 2)(27.3 \mathrm{eV})$, (b) streaky $c(2 \times 2)(27.2 \mathrm{eV})$, (c) $c(2 \times 8)(25.9 \mathrm{eV}),(\mathrm{d}) c(2 \times 10)(26.0 \mathrm{eV})$. The LEED pattern in (a) was obtained from the sample region depicted in Fig. 5(b).

dition to very diffuse intensity in the $\left(\frac{1}{2}, \frac{1}{2}\right)$ and equivalent positions (the $\frac{1}{2}$-order positions) suggestive a $c(2 \times 2)$ diffraction pattern. The diffuse intensity near the $\frac{1}{2}$-order positions arises due to critical fluctuations of the clean $\mathrm{Mo}(100)$ $c(7 \sqrt{2} \times \sqrt{2}) R 45^{\circ}$ surface reconstruction which has its ordering critical temperature below room temperature. ${ }^{35}$ This critical scattering vanishes rapidly with increasing temperature above the critical temperature and was completely absent in all of the experiments described below that were carried out at elevated temperatures. Since the clean surface reconstruction is easily quenched by small amounts of contaminants, our observation of critical scattering is a good indication of sample cleanliness.

Interaction of Pd with the Mo(100) surface was studied primarily by deposition onto the surface at fixed temperature, and to a lesser extent by postannealing films at the growth temperature or to higher temperature. The most notable result was the development of distinct $c(2 \times 2)$ and $c(2 \times n)$ diffraction patterns (Fig. 3). Deposition in the temperature range 780-980 K resulted initially in the appearance of diffuse LEED intensity at the $c(2 \times 2) \frac{1}{2}$-order positions, which developed dramatically into sharp and intense spots with further increase of Pd coverage [Fig. 3(a)]. The $c(2 \times 2)$ diffraction pattern induced by $\mathrm{Pd}$ on $\mathrm{Mo}(100)$ resembles the intense metal-induced $c(2 \times 2)$ patterns that had been observed previously on the W(100) (Refs. 12 and 13) and Mo(100) (Refs. 14 and 15) surfaces. Since it is wellestablished that the ideal metal coverage for those $c(2 \times 2)$ structures is $0.5 \mathrm{ML}$, we adopt this coverage assignment here for the $\mathrm{Mo}(100)-\mathrm{Pd} c(2 \times 2)$ structure. All coverages and deposition rates quoted in the following discussion are made with reference to this assignment.

Deposition of additional material in this temperature range, $780-980 \mathrm{~K}$, resulted in a decrease of the intensity at the $\frac{1}{2}$-order spot positions and at the same time caused the $c(2 \times 2)$ diffraction spots to streak in the [001] and [010] directions. The streaking became more pronounced with increasing coverage until only crosslike features remained centered at the $\frac{1}{2}$-order positions [Fig. 3(b)]. With further deposition, the $\frac{1}{2}$-order crosses gradually disappeared while a

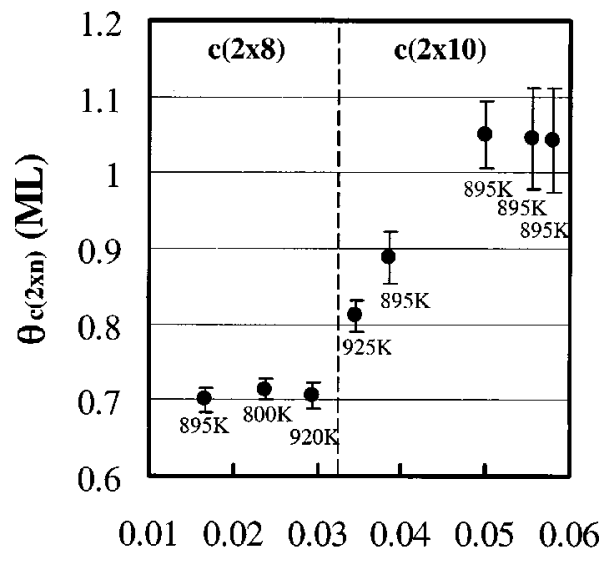

\section{Deposition Rate (ML/min)}

FIG. 4. Mo(100)-Pd $c(2 \times n)$ periodicity and completion coverage vs deposition rate. The deposition temperature is indicated adjacent to each data point.

distinct quartet of spots centered at the $\frac{1}{2}$-order positions developed at the same time in their place [Figs. 3(c) and 3(d)]. These and other new spots that appeared simultaneously were indexed $\left(\frac{1}{2}, \frac{1}{2} \pm(2 m+1) / n\right)$ and $\left(\frac{1}{2} \pm(2 m+1) / n, \frac{1}{2}\right)$ and equivalent positions (where $m$ and $n$ are integers and $m=0, \pm 1, \pm 2, \ldots)$. Concurrent with the appearance of these superstructure spots was the development of additional spots along the [001] and [010] vectors joining nearest integerorder spots, e.g., $(0,2 m / n)$ and $(2 m / n, 0)$. The resultant LEED pattern is readily seen to be the superposition of two degenerate $c(2 \times n)$ structures with $n$-fold periodicity in the orthogonal [001] and [010] directions, respectively. These degenerate structures are related by a rotation of $90^{\circ}$ about the surface normal. The $c(2 \times n)$ diffraction persisted without any dramatic change during additional deposition up to a total coverage of 2.5 ML. Higher coverages were not studied.

Only two $c(2 \times n)$ periodicities, $n=8$ and $n=10$, were found in our work. The key factor that determined the selection of periodicity was the deposition rate. Figure 4 summarizes the $c(2 \times n)$ periodicities and apparent coverages required to completely form these structures, $\theta_{c(2 \times n)}$, as a function of the deposition rate. The deposition temperatures are indicated by the notation adjacent to each data point. A clear boundary is seen separating deposition rates that led to $c(2 \times 8)$ and $c(2 \times 10)$ structures. The $c(2 \times 8)$ structure was observed during "slow" growth at rates below approximately $0.033 \mathrm{ML} / \mathrm{min}$. The formation of the $c(2 \times 8)$ was apparently complete at a coverage of $\theta_{c(2 \times 8)}=0.70 \mathrm{ML}$ for all deposition rates that led to this periodicity. At that point, sharp, instrument-limited superstructure LEED spots were observed that indicated that the $c(2 \times 8)$ structure was very well ordered as-grown [Fig. 3(c)]. When the deposition rate exceeded about $0.033 \mathrm{ML} / \mathrm{min}$, a number of changes occurred, most notably the formation of a $c(2 \times 10)$ instead of $c(2 \times 8)$ structure [Fig. 3(d)]. The completion coverage, $\theta_{c(2 \times 10)}$, also increased sharply with deposition rate until a saturation value of about $1 \mathrm{ML}$ was reached at the highest deposition rates studied (Fig. 4). In addition, the $c(2 \times 10)$ superstructure spots were streaky in the $n$-fold periodic di- 
rection, and their streakiness was more pronounced at higher deposition rates. This indicates that the $c(2 \times 10)$ structure was somewhat less well ordered than the $c(2 \times 8)$. Consequently, there was greater uncertainty in the determination of $\theta_{c(2 \times 10)}$, as indicated by the error bars in Fig. 4. Ordering of the $c(2 \times 10)$ structure could be improved markedly by postannealing at the deposition or higher temperature. For example, the $c(2 \times 10)$ LEED pattern in Fig. 3(d) was obtained after depositing $1.2 \mathrm{ML}$ of $\mathrm{Pd}$ at $895 \mathrm{~K}$ at a rate of 0.05 $\mathrm{ML} / \mathrm{min}$ followed by annealing at $950 \mathrm{~K}$ for $10 \mathrm{~min}$. Despite the improvement realized by postannealing, some slight streaking or elongation of the $c(2 \times 10)$ diffraction spots remains.

Most of the data points in Fig. 4 fall into a narrow $\pm 15 \mathrm{~K}$ range of $910 \mathrm{~K}$. This raises the question of whether the boundary between $c(2 \times 8)$ and $c(2 \times 10)$ depicted in Fig. 4 depends upon deposition temperature in a wider range. While not addressed extensively in our work, the data point for $0.023 \mathrm{ML} / \mathrm{min}$ at $800 \mathrm{~K}$ provides an answer to this question. A very well-ordered $c(2 \times 8)$ structure was obtained at this deposition rate although the deposition temperature was significantly lower than the neighboring data points in the $c(2 \times 8)$ regime. This leads us to believe that the deposition temperature will not dramatically influence the periodicity selection.

The structural evolution of Pd films that were deposited at temperatures outside the range 780-980 K differed somewhat from the sequence described above. Although intense $c(2 \times 2)$ diffraction spots formed and then became streaky with increasing Pd coverage during deposition at $1035 \mathrm{~K}$, similar to the sequence below $980 \mathrm{~K}$, the $c(2 \times n)$ structure did not develop at this elevated temperature. Instead, the surface transformed by way of a diffuse $c(2 \times 2)$ back to a $(1 \times 1)$ condition at approximately $1.5 \mathrm{ML}$. Deposition at higher temperatures was not explored. At the lowertemperature side, deposition at $580 \mathrm{~K}$ resulted in a $c(2 \times 2)$ diffraction pattern that was noticeably much weaker and more diffuse than observed above $780 \mathrm{~K}$. It was also not possible to distinguish a $c(2 \times n)$ structure at higher coverage when deposition was carried out at $580 \mathrm{~K}$. Only a (1 $\times 1)$ diffraction pattern was observed during Pd deposition at room temperature. A weak but sharp $c(2 \times 8)$ diffraction pattern developed when a 0.7 ML pseudomorphic film deposited at room temperature was annealed above $780 \mathrm{~K}$. The key point that we wish to make here is that the $c(2 \times 2)$ and $c(2 \times n)$ structures do not form well if a deposition temperature below about $780 \mathrm{~K}$ is used or if films deposited at room temperature are annealed below $780 \mathrm{~K}$. There is also clear evidence of an upper-temperature limit to the stability of the $c(2 \times n)$ structures. The behavior of $\mathrm{Pd} / \mathrm{Mo}(100)$ in the higher-temperature regime is discussed further in Sec. III C.

\section{B. Morphology}

Complementary LEEM investigations of Pd film growth revealed that a remarkable surface morphological change accompanied the formation of the $c(2 \times 2)$ and $c(2 \times n)$ structures. The nucleation and growth of a large number of islands were seen with real-time LEEM observations during Pd deposition in the temperature range 780-980 K (Figs. 5 and 6). Islands were observed to nucleate on the largest ter-
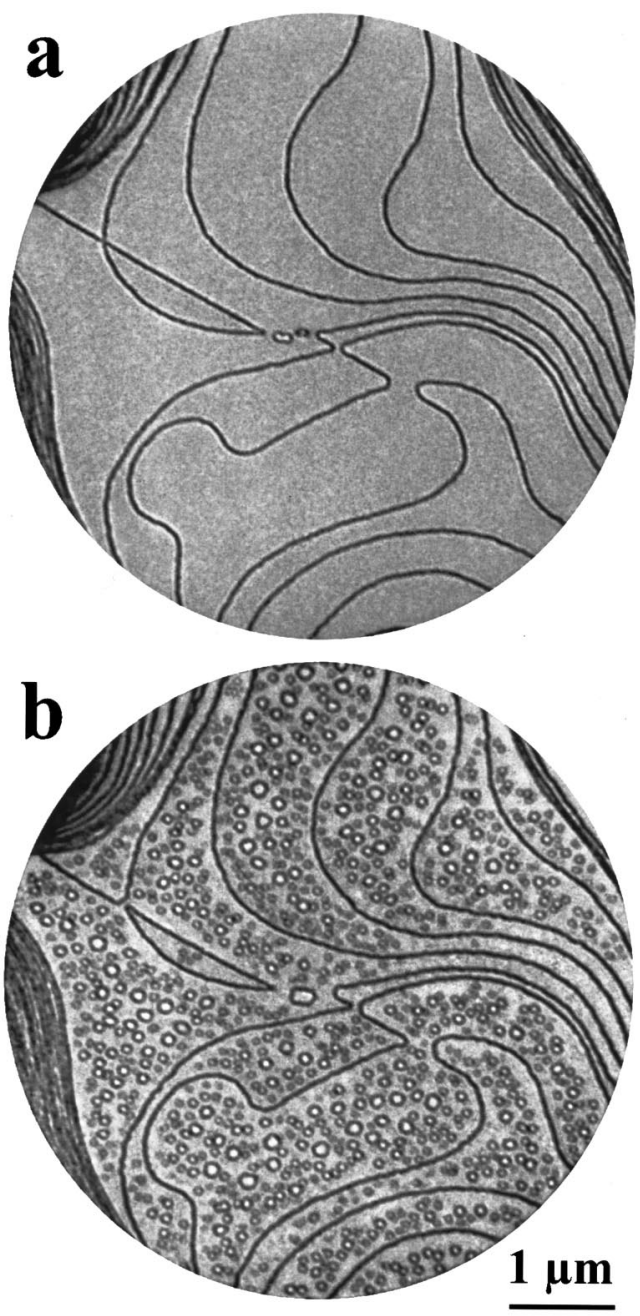

FIG. 5. LEEM images of (a) clean Mo(100) and (b) Mo(100)-Pd $c(2 \times 2)$. The imaging energy was $4.8 \mathrm{eV}$. Dark lines indicate monatomic height steps. The LEED pattern in Fig. 3(a) was obtained from the sample region depicted in (b).

races (e.g., terraces of the size depicted in Fig. 6) already after a deposition of below 0.1 ML. Island nucleation on smaller terraces was delayed somewhat due to the close proximity of step sinks. The island size and also number steadily increased until a coverage, $\theta=0.5 \mathrm{ML}$, corresponding to the best developed $c(2 \times 2)$ structure was reached [Fig. 5(b)]. Deposition of additional material leading to the formation of the $c(2 \times n)$ structures [Fig. 6(b)] and beyond effected very little further change of the morphology.

Identification of step height and sense-step-up versus step-down directions - can be easily made with LEEM by inspection of step-contrast interference fringes at various imaging energies. ${ }^{34}$ In particular, a bright interference fringe appears at the upper side of steps at the energy used to obtain the images in Figs. 5 and 6. Using this sensitivity to the nature of steps, we have determined that the islands formed during Pd deposition are positive, as opposed to negative or vacancy islands, and with single-atom height. This allows for the determination of the amount of material contained in the islands just by examining their area. 

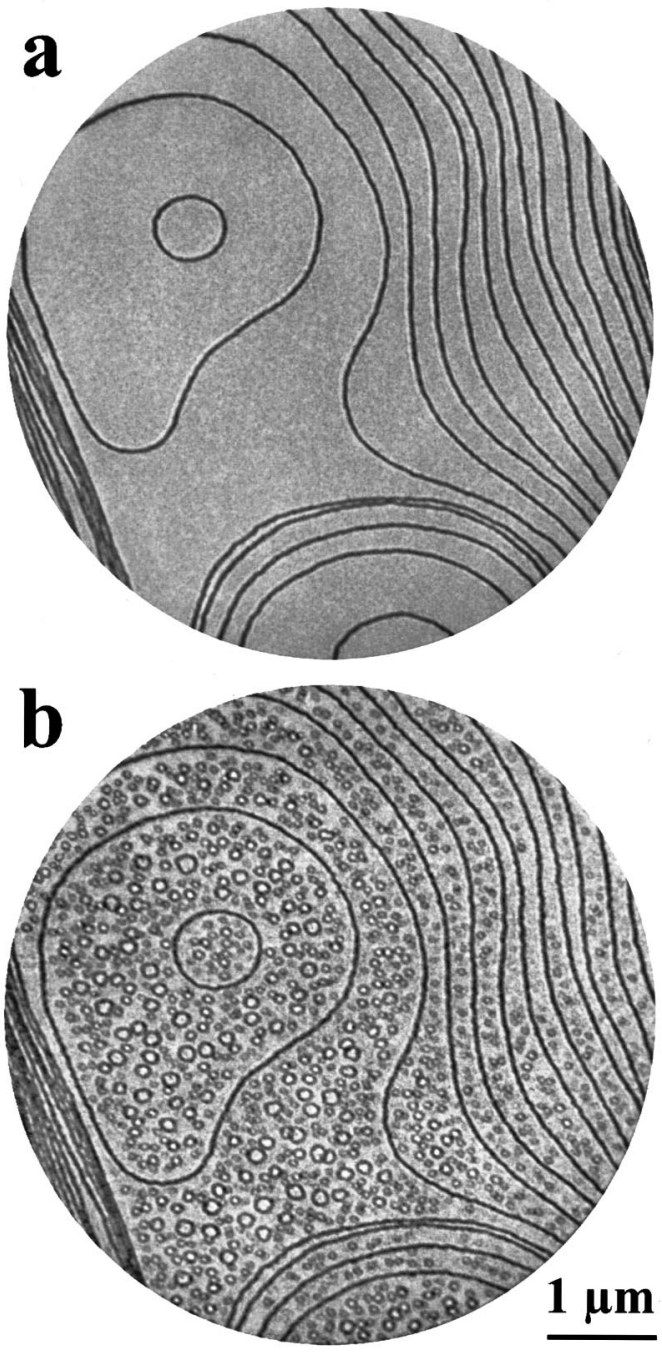

FIG. 6. LEEM images of (a) clean Mo(100) and (b) Mo(100)-Pd $c(2 \times 10)$. The imaging energy was $4.8 \mathrm{eV}$. Dark lines indicate monatomic height steps.

\section{Faceting}

Stimulated by the theoretical predictions outlined above, an additional aim of our experiments was to search for evidence of Pd-induced faceting of the $\mathrm{Mo}(100)$ surface. On the $\mathrm{W}$ and Mo(111) surfaces, faceting occurs when $3 \mathrm{ML}$ (equivalently $1 \mathrm{PML}$ ) pseudomorphic Pd films are briefly annealed above $700 \mathrm{~K}^{6,7}$ Annealing of thicker films also causes faceting but with material in excess of $3 \mathrm{ML}$ collected into isolated three-dimensional Pd clusters on top of the faceted surface. This faceting is quite easily identifiable with LEED by the appearance of additional spots that move rapidly between integer-order spots as the incident electron energy is varied. Reiterating the predictions for $\mathrm{Pd} / \mathrm{Mo}(100)$, the substrate is potentially unstable with respect to faceting to $\{211\}$ in the presence of a 2 ML pseudomorphic film. In our experiments, however, the $c(2 \times n)$ structure was found to persist in the temperature range 780-980 $\mathrm{K}$ during continuous Pd deposition up to the maximum coverage studied, 2.5 ML. No evidence of the transformation from $c(2 \times n)$ to pseudomorphic structure or faceting was seen in LEED in this coverage and temperature range. Likewise, postannealing $c(2 \times n)$ films at the growth or higher temperature did not result in a pseudomorphic film or any faceting behavior. The only effect of postannealing was to sharpen up the $c$ ( 2 $\times 10)$ superstructure diffraction spots, which signaled an improvement of ordering, while little effect was seen on $c(2$ $\times 8)$ films that were already well-ordered as-grown. Also, no evidence of three-dimensional Pd clustering was seen within the resolution limit of LEEM for any coverage or thermal treatment. Little work was carried out above $980 \mathrm{~K}$. In one instance, $c(2 \times 10)$ diffraction from a $2.5 \mathrm{ML}$ film was converted to a diffuse $c(2 \times 2)$ when the sample temperature was raised to $1050 \mathrm{~K}$. The diffraction pattern gradually reverted to $(1 \times 1)$ as the temperature was raised to $1150 \mathrm{~K}$. No evidence for faceting was seen as a result of this thermal treatment and neither the $c(2 \times n)$ or $c(2 \times 2)$ structures returned upon cooling to room temperature. While it is conceivable that these changes were due to the irreversible formation of a pseudomorphic film, they were also possibly the result of disordering of the $c(2 \times n)$ structure or the loss of Pd either through desorption or diffusion to other parts of the sample. These points will be discussed again in more detail in the following section.

\section{DISCUSSION}

First-principles calculations demonstrate that $0.5 \mathrm{ML}$ of $\mathrm{Pd}$ on $\mathrm{Mo}(100)$ is energetically more stable in a $c(2 \times 2)$ substitutional alloy than in a $c(2 \times 2)$ overlayer configuration. It is also notable that the energy of the $c(2 \times 2)$ alloy (configuration $A$ ) at $0.5 \mathrm{ML}$ is clearly below the line in Fig. 2 that connects the point corresponding to a pseudomorphic layer (configuration $C$ ) at $1 \mathrm{ML}$ and the point corresponding to a clean surface at $0 \mathrm{ML}$. Just as importantly, the $c(2$ $\times 2$ ) overlayer model (configuration $B$ ) at $0.5 \mathrm{ML}$ lies above this line. This means that the $c(2 \times 2)$ overlayer structure would be preempted by the formation of a heterogeneous system at 0.5 ML coverage in which half the surface is covered with islands of 1 ML Pd coverage and the other half is exposed, clean $\mathrm{Mo}(100)$. This also means that the $c(2 \times 2)$ alloy structure is energetically favored compared to the heterogeneous system and will form provided the energy barrier to exchange Pd for surface Mo atoms can be overcome. Our experiment shows that the Pd-induced $c(2 \times 2)$ diffraction pattern is very weak and diffuse and thus that the kinetic barrier to exchange can hardly be overcome during deposition at $580 \mathrm{~K}$. A similar conclusion is reached about roomtemperature deposited films that are annealed to $580 \mathrm{~K}$. These results are consistent with earlier observations of "fuzzy" $c(2 \times 2)$ diffraction from $\mathrm{Pd} / \mathrm{Mo}(100)$ below 600 $\mathrm{K} .{ }^{20}$ On the other hand, the intense $c(2 \times 2)$ diffraction pattern that is induced by Pd above $780 \mathrm{~K}$ is compelling evidence, in light of our theoretical calculations, of the formation of the surface alloy structure shown in Fig. 1(a)

LEEM observations of morphological changes that occur during Pd deposition at elevated temperature are also consistent with the formation of the $c(2 \times 2)$ surface alloy. First of all, the monatomic height islands that appeared during the formation of the Pd-induced superstructures were indistinguishable from the neighboring terraces on the basis of LEEM diffraction contrast. They could be detected only by LEEM step contrast, which is a phase-contrast mechanism that provides no information about local geometric structure. 
This means that the islands have the same composition and structure as the surrounding terrace, which would not be the case if the islands were composed purely of Pd in $c(2 \times 2)$ or pseudomorphic overlayer arrangements. Second, overlayer islands composed purely of Pd might be expected to continue to grow, coalesce and ultimately return the surface to a flat state upon the completion of 1 layer. Contrary to this expectation, the Pd-induced islands ceased growing at about the same time that the $c(2 \times 2)$ diffraction became most intense, or shortly thereafter. The surface never regained a flat appearance even after well over $1 \mathrm{ML}$ of Pd had been deposited. These observations are inconsistent with Pd overlayer structures and are instead more easily understood in terms of the $c(2 \times 2)$ surface alloy. In order to form the alloy structure, $\mathrm{Pd}$ atoms must exchange for Mo atoms in the topmost layer on terraces. The displaced Mo atoms combine with deposited Pd adatoms to form $c(2 \times 2)$ alloy islands on top of the terraces. However, the supply of Mo atoms is limited because the $c(2 \times 2)$ alloy does not penetrate beyond the first layer. The entire surface will be completely covered with $c(2 \times 2)$ alloy-half of this residing in the original terrace level and the other half present in the form of islands one layer up-when only $0.25 \mathrm{ML}$ of Mo has been supplied from terraces. In other words, islands stop growing at the point that they cover half of the surface. This occurs at the same Pd coverage, $\theta_{c(2 \times 2)}=0.5 \mathrm{ML}$, for which the $c(2 \times 2)$ diffraction pattern is best developed. LEEM observations of island growth and morphology are in qualitative agreement with this scenario.

LEEM also reveals that many very small and barely resolveable islands appear between larger islands in the later stages of the $c(2 \times 2)$ alloy formation, and that there are slightly more of these very small islands on the $c(2 \times n)$ surface than on the $c(2 \times 2)$ surface. Otherwise, the $c(2$ $\times 2)$ and $c(2 \times n)$ surfaces can hardly be distinguished by LEEM. The fact that little morphological change is associated with the transformation from $c(2 \times 2)$ to $c(2 \times n)$ structures is a good indication that this transformation mostly involves minor local rearrangements. Another key observation for understanding the $c(2 \times n)$ structure is the continuous evolution of the $c(2 \times n)$ LEED pattern, by way of a streaky $c(2 \times 2)$, from the sharp $c(2 \times 2)$ diffraction pattern. This sequence suggests that the $c(2 \times 2)$ alloy structure develops an irregular arrangement of antiphase domain walls in the [001] or [010] directions whose spacing gradually become ordered to $n$-fold periodicity. The proposed $c(2 \times n)$ surface alloy structure is shown in Fig. 1(b). In this structure, domain walls consist of fully Pd-substituted [001] rows of atoms in the topmost layer. These Pd rows separate domains that retain the local $c(2 \times 2)$ alloy structure but that exist in antiphase relationship by a shift of one lattice spacing in the [001] direction. The primitive vectors and centered unit cell are shown in Fig. 1(b). In the equivalent $90^{\circ}$-rotated domain, the fully Pd-substituted rows run along the [010] direction. Actually, the domain walls in the $c(2 \times n)$ structure need not be exactly as shown in Fig. 1(b). The key point is that they should have a higher Pd content than the $c(2 \times 2)$ structure itself, this condition being imposed by the fact that the $c(2$ $\times n$ ) structure forms only if Pd is deposited in excess of the ideal $c(2 \times 2)$ coverage. The exchange of additional Pd for Mo atoms in the topmost layer may be responsible for the slightly larger number of very small islands in LEEM images of the $c(2 \times n)$ surface compared to the $c(2 \times 2)$ surface.

Alternatively, the $c(2 \times n)$ structure may be related to one of the bulk Pd-Mo compounds that have been reported. ${ }^{36}$ $\mathrm{Pd}_{2} \mathrm{Mo}$, in particular, is stable in a narrow $\mathrm{Pd}$ concentration range that is intermediate between those of the $c(2 \times 8)$ and $c(2 \times 10)$ structures. ${ }^{37}$ However, this and other bulk compounds with $\mathrm{Pd}$ concentration that span the $c(2 \times n)$ concentration have fcc structure with lattice constant of approximately $3.9 \AA$ A. There is no clear relationship between this structure and cell dimension and the $c(2 \times n)$ periodicity. Therefore, it seems unlikely that the surface alloys are twodimensional realizations of the bulk compounds.

Growth of the $c(2 \times 8)$ layer at low deposition rates is believed to take place close to equilibrium because it is very well-ordered as-grown. This has the implication that the completion coverage of the $c(2 \times 8)$ structure, $\theta_{c(2 \times 8)}$ $=0.70 \mathrm{ML}$, is probably fairly close to the true Pd content of this alloy structure. The experimental value is in fair agreement although a little larger than the Pd composition, 0.625 ML, of the $c(2 \times 8)$ alloy structure shown in Fig. 1(b). This small discrepancy may be due to a systematic error in our coverage assignment. On the other hand, the excessive amount of material deposited in experiment may be an indication that there is still a small kinetic limitation to the formation of the $c(2 \times 8)$ alloy structure, probably related to the exchange process. This limitation is clearly more severe when the deposition rate exceeds $0.033 \mathrm{ML} / \mathrm{min}$. At higher deposition rates, $\mathrm{Pd}$ atoms impinge the surface faster than they can become incorporated into the $c(2 \times 8)$ structure by exchange with Mo atoms in the top layer, and the alloy layer gets covered over more rapidly by excess Pd. Consequently, the long-range ordering process is hindered and the system settles into a $c(2 \times 10)$ structure that is somewhat less ordered and with lower actual Pd content than the $c(2 \times 8)$. There is an increasing tendency to exceed or overshoot the coverage necessary to form the $c(2 \times 10)$, i.e., $\theta_{c(2 \times 10)}$ increases, as the deposition rate increases. The apparent $c(2$ $\times 10$ ) coverage rises to about $1 \mathrm{ML}$ although the coverage required to form a $c(2 \times 10)$ of the type shown in Fig. 1(b) is only $0.60 \mathrm{ML}$. Interestingly, a $c(2 \times 6)$ diffraction pattern was also observed previously when a pseudomorphic $1 \mathrm{ML}$ Pd film was deposited at $150 \mathrm{~K}$ and subsequently annealed to $1300 \mathrm{~K}^{20}{ }^{20}$ The model shown in Fig. 1(b) can easily account for this periodicity by appropriate adjustment of the domain wall spacing.

Theory shows that the $\mathrm{Pd} / \mathrm{Mo}(100)$ system can continue to lower its energy with increasing coverage by assuming pseudomorphic configurations at 1 and 2 ML (configurations $C$ and $F$, respectively), and may be unstable with respect to faceting when covered by a 2 ML-thick pseudomorphic Pd film. According to Fig. 2, however, one pseudomorphic Pd ML (configuration $C$ at $1 \mathrm{ML}$ ) lies approximately along the line joining the $c(2 \times 2)$ alloy (configuration $A$ at $0.5 \mathrm{ML}$ ) and the $c(2 \times 2)$ alloy covered by 1 pseudomorphic Pd ML (configuration $E$ at $1.5 \mathrm{ML}$ ). This means that the pseudomorphic Pd monolayer (configuration $C$ ) is energetically very close to a heterogeneous system at $1 \mathrm{ML}$ coverage in which a complete $c(2 \times 2)$ surface alloy layer is half-covered with pseudomorphic Pd islands (a 50:50 mixture of configurations $A$ and $E$ ). Thus, the thermodynamic driving force to form the 
pseudomorphic $\mathrm{Pd}$ monolayer (configuration $C$ ) is small in relation to burying the $c(2 \times 2)$ alloy under additional $\mathrm{Pd}$. However, the real situation is a little more complicated than this. Experiment shows that the $\mathrm{Pd} / \mathrm{Mo}(100)$ system assumes some fairly complex $c(2 \times n)$ configurations after the completion of the $c(2 \times 2)$ surface alloy at $0.5 \mathrm{ML}$ coverage. These complex surface alloy configurations persist to higher coverage and apparently preempt the formation of pseudomorphic films (configurations $C$ and $F$ ) between 780-980 K. Our LDA calculations are limited to simple pseudomorphic or $c(2 \times 2)$ configurations. At this point, therefore, we do not know whether the complex alloy configurations observed in the experiment have lower free energy than the higher coverage pseudomorphic configurations or whether the kinetic barrier to the formation of the pseudomorphic films is prohibitive in this temperature range.

Possible evidence for the transformation from surface alloy to pseudomorphic structure came during continuous deposition and postannealing above $980 \mathrm{~K}$. To repeat what was reported above (see Sec. III C), $c(2 \times 10)$ diffraction from a $2.5-\mathrm{ML}$ film deposited below $980 \mathrm{~K}$ was converted during annealing to $1050 \mathrm{~K}$ to a diffuse $c(2 \times 2)$ structure, and the surface gradually returned to $(1 \times 1)$ as the temperature was raised to $1150 \mathrm{~K}$. In a separate experiment (see Sec. III A), the system returned to a $(1 \times 1)$ configuration after passing through a $c(2 \times 2)$ structure during continuous deposition of more than 1.5 ML at $1035 \mathrm{~K}$. These changes were probably not due to desorption since desorption of Pd films thicker than $1 \mathrm{ML}$ on $\mathrm{Mo}(100)$ was reported to begin at about $1250 \mathrm{~K} .{ }^{20}$ This is well above the temperature where the $c(2 \times n)$ and $c(2 \times 2)$ diffraction began to disappear in our experiments. Another possible explanation for the reappearance of the $(1 \times 1)$ diffraction at higher $\mathrm{Pd}$ coverages above $1035 \mathrm{~K}$ is disordering of the surface alloy structure. Lateral disordering confined to the interface between Mo and thick Pd films does not seem likely. A more likely disordering mechanism is bulk alloying that occurs as the result of interdiffusion of Mo into the Pd film. Interdiffusion of Pd in Mo can be ruled out on the basis of our theoretical calculations. Alloying of W(100) (Refs. 16 and 18), W(110) (Ref. 4), and Mo(110) (Ref. 4) with thick Pd films is known to start at 500, 800, and $770 \mathrm{~K}$, respectively. Evidence of alloying of $\mathrm{Mo}(100)$ with thick $\mathrm{Pd}$ films (>2 ML) above $870 \mathrm{~K}$ has also been reported in the absence of the $c(2 \times n)$ structures. $^{20}$ Interdiffusion may be suppressed until the higher temperatures in our work due to the presence of the ordered two-dimensional alloy structures at the interface.

Since pseudomorphic films could not be realized by continuous deposition at elevated temperature or by postannealing films in our work, it was not possible to fully test theoretical predictions of faceting. In previous work on $\mathrm{Pd} /$ Mo(100), pseudomorphic films of thickness sufficient to induce faceting could be grown at $150 \mathrm{~K} .{ }^{20}$ Apparently no evidence of faceting was found in subsequent annealing experiments up to the desorption temperature. As noted in Sec. II, the potential for a faceting instability in the $\mathrm{Pd} / \mathrm{Mo}(100)$ system is suggested by thermodynamic considerations. This prediction does not take into account the kinetic barrier to facet nucleation and mass transport. The situation is further complicated by the presence of complex $c(2 \times n)$ surface alloy structures. Nevertheless, it is still interesting because we now have a case in which an "atomically smooth" (100)oriented surface can in principle become unstable (or marginally stable) with regard to faceting to other orientations, while the common wisdom is that only "atomically rough", surfaces would facet.

\section{SUMMARY}

The interaction of Pd with the Mo(100) surface at elevated temperature has been found in this work to generate an intense $c(2 \times 2)$ diffraction pattern. First-principles calculations demonstrate that a $c(2 \times 2)$ substitutional surface alloy is energetically favored compared to overlayer structures at $0.5-\mathrm{ML}$ coverage. Charge transfer from the Mo substrate to the $\mathrm{Pd}$ adatoms is responsible for stabilizing a $c(2 \times 2)$ vacancy array, into which the $\mathrm{Pd}$ atoms are incorporated. Morphological changes that accompany the formation of the $\mathrm{Mo}(100)-\mathrm{Pd} c(2 \times 2)$ structure are also consistent with the exchange of $\mathrm{Pd}$ for $\mathrm{Mo}$ surface atoms. The $\mathrm{Pd}$-induced $\operatorname{Mo}(100) c(2 \times 2)$ structure is therefore closely related to the $c(2 \times 2)$ substitutional surface alloy structures that are produced by annealing $0.5 \mathrm{ML}$ of $\mathrm{Cu}, \mathrm{Au}, \mathrm{Ag}$, or $\mathrm{Pt}$ on the $\mathrm{W}(100)$ surface. On the basis of our results, it is therefore necessary to reinterpret the $\mathrm{Au}$ - and $\mathrm{Cu}$-induced $\mathrm{Mo}(100)$ $c(2 \times 2)$ structures that were considered previously to be overlayer structures. Our results also stimulate a closer examination of the interaction of Pd with the W(100) surface. Evidence of a $c(2 \times 2)$ surface alloy may be found in this system if deposition conditions similar to those used in the present work are employed.

In addition to the $c(2 \times 2)$ at $0.5 \mathrm{ML}$ coverage, we have also discovered that $\mathrm{Pd}$ induces $c(2 \times n)$ structures $(n$ $=8,10)$ on $\operatorname{Mo}(100)$ at higher coverages. These structures have not been observed on Mo or W(100) surfaces in contact with metal overlayers before. Experimental observations suggest that the transformation from $c(2 \times 2)$ to $c(2 \times n)$ structure fundamentally involves local rearrangements in the alloy layer. A model that incorporates antiphase domain walls with high $\mathrm{Pd}$ content into the $c(2 \times 2)$ surface alloy structure is proposed to explain the $c(2 \times n)$ structures. The kinetics of Pd exchange for Mo surface atoms has been found to play an important role in $n$-fold periodicity selection.

Theoretical calculations indicate that a pseudomorphic Pd overlayer is the most stable configuration at $1 \mathrm{ML}$ coverage and that the system can continue to lower its energy by retaining a pseudomorphic configuration at $2 \mathrm{ML}$ coverage. Potential instability with respect to faceting to $\{211\}$ orientations is also predicted if pseudomorphic $2 \mathrm{ML}$ films can be realized, in analogy to metal-induced faceting of the Mo and $\mathrm{W}(111)$ surfaces. However, the $c(2 \times n)$ structure is found experimentally to be stable between Mo and thicker Pd overlayers in the elevated temperature range that faceting is expected to occur. Since the calculations were limited to simple pseudomorphic and $c(2 \times 2)$ configurations, it is not known whether the $c(2 \times n)$ structure is energetically more stable or whether the kinetic barrier to formation of pseudomorphic films is prohibitive. Even if pseudomorphic $2 \mathrm{ML}$ films were not preempted by the $c(2 \times n)$ alloy at elevated temperature, the thermodynamic driving force for faceting is rather small 
for $\mathrm{Pd} / \mathrm{Mo}(100)$ and may not be sufficient to overcome a large kinetic barrier to facet nucleation and massive mass transport. It would therefore be interesting to explore whether other metals induce similar complex surface alloy structures or faceting on the $\operatorname{Mo}(100)$ surface in the future.

\section{ACKNOWLEDGMENTS}

This work was supported by the Hong Kong Research Grant Council under Grants Nos. HKUST690/96P and HKUST6128/98P.
*Corresponding author. Email: phaltman@ust.hk.

${ }^{1}$ J. G. Che, C. T. Chan, C. H. Kuo, and T. C. Leung, Phys. Rev. B 57, 1875 (1998).

${ }^{2}$ H. Landolt and R. Börnstein, Numerical Data and Functional Relationships in Science and Technology (Springer, Berlin, 1987), Vol. 24, Subvolume D, p. 352.

${ }^{3}$ Metal overlayers on $\mathrm{W}$ and Mo surfaces have been studied extensively. For an early review, see E. Bauer, in The Chemical Physics of Solid Surfaces and Heterogeneous Catalysis, edited by D. A. King, and D. P. Woodruff (Elsevier, Amsterdam, 1984), Vol. 3, Part B.

${ }^{4}$ For Pd on W and Mo(110), see specifically W. Schlenk and E. Bauer, Surf. Sci. 93, 9 (1980); Ch. Park, E. Bauer, and H. Poppa, ibid. 154, 37 (1985); E. Bauer, H. Poppa, G. Todd, and P. R. Davis, J. Appl. Phys. 48, 3773 (1977).

${ }^{5}$ J. Guan, R. A. Campbell, and T. E. Madey, Surf. Sci. 341, 311 (1995); T. E. Madey, J. Guan, C. H. Nien, C. Z. Dong, H. S. Tao, and R. A. Campbell, Surf. Rev. Lett. 3, 1315 (1996).

${ }^{6}$ K. J. Song, C. Z. Dong, and T. E. Madey, Langmuir 7, 3019 (1991); C. Z. Dong, L. Zhang, U. Diebold, and T. E. Madey, Surf. Sci. 322, 221 (1995); K. J. Song, J. C. Lin, M. Y. Lai, and Y. L. Wang, ibid. 327, 17 (1995); H. S. Tao, C. H. Nien, T. E. Madey, J. E. Rowe, and G. K. Wertheim, ibid. 357/358, 55 (1996); C. H. Nien and T. E. Madey, ibid. 380, L527 (1997).

${ }^{7}$ C. H. Nien, T. E. Madey, Y. W. Tai, T. C. Leung, J. G. Che, and C. T. Chan, Phys. Rev. B 59, 10355 (1999).

${ }^{8}$ J. G. Che, C. T. Chan, C. H. Kuo, and T. C. Leung, Phys. Rev. Lett. 79, 4230 (1997).

${ }^{9}$ J. G. Che and C. T. Chan, Surf. Sci. 401, L432 (1998).

${ }^{10}$ J. J. Kolodziej, K. Pelhos, I. M. Abdelrehim, J. W. Keister, J. E. Rowe, and T. E. Madey, Prog. Surf. Sci. 59, 117 (1998).

${ }^{11}$ E. Bauer, H. Poppa, G. Todd, and P. R. Davis, J. Appl. Phys. 48, 3773 (1977).

${ }^{12}$ G. A. Attard and D. A. King, Surf. Sci. 188, 589 (1987); 222, 351 (1989); 222, 360 (1989); 223, 1 (1989); P. Hu, A. Wander, L. Morales de la Garza, M. P. Bessent, and D. A. King, ibid. 286, L542 (1993).

${ }^{13}$ R. W. Judd, M. A. Reichelt, E. G. Scott, and R. M. Lambert, Surf. Sci. 185, 515 (1987).

${ }^{14}$ F. Soria and H. Poppa, J. Vac. Sci. Technol. 17, 449 (1980).

${ }^{15}$ J. R. Cerda, F. Soria, F. J. Palomares, and P. L. de Andres, Surf. Sci. 269/270, 713 (1992).

${ }^{16}$ S. Prigge, H. Roux, and E. Bauer, Surf. Sci. 107, 101 (1981).

${ }^{17}$ P. J. Berlowitz and D. W. Goodman, Langmuir 4, 1091 (1988).

${ }^{18}$ H. Woormeester, E. Hüger, and E. Bauer, Phys. Rev. Lett. 77, 1540 (1996); Phys. Rev. B 54, 17108 (1996).

${ }^{19}$ E. Bauer (private communication).
${ }^{20}$ J. M. Heitzinger, S. C. Gebhard, D. H. Parker, and B. E. Koel, Surf. Sci. 260, 151 (1992).

${ }^{21}$ See, e.g., Theory of the Inhomogeneous Electron Gas, edited by N. H. March and S. Lundqvist (Plenum, New York, 1983), and references therein; D. M. Ceperly and B. J. Alder, Phys. Rev. Lett. 45, 566 (1980); J. P. Perdew and A. Zunger, Phys. Rev. B 23, 5048 (1981).

${ }^{22}$ G. B. Bachelet and M. Schluter, Phys. Rev. B 25, 2103 (1982).

${ }^{23}$ C. Elsaesser, N. Takeuchi, K. M. Ho, C. T. Chan, P. Braun, and M. Fahnle, J. Phys.: Condens. Matter 2, 4371 (1990); K. M. Ho, C. Elsaesser, C. T. Chan and M. Fahnle, ibid. 4, 5189 (1992).

${ }^{24}$ C. T. Chan, K. P. Bohnen, and K. M. Ho, Phys. Rev. B 47, 4771 (1993).

${ }^{25}$ Our calculations allow for a $(\sqrt{2} \times \sqrt{2}) R 45^{\circ}$, i.e., $c(2 \times 2)$ DebeKing type reconstruction. The experimentally observed reconstruction of $\mathrm{Mo}(100)$ actually has an extra surface modulation making it $c(7 \sqrt{2} \times \sqrt{2}) R 45^{\circ}$ [see, e.g., R. S. Daley, T. E. Felter, M. K. Hildner, and P. J. Estrup, Phys. Rev. Lett. 70, 1295 (1993)]. The surface energies in such reconstructions are of the order of mRy per surface atom lower than the $p(1 \times 1)$ unreconstructed surface, and are very small compared with the energy scale we are comparing.

${ }^{26}$ See, e.g., J. G. Che, Z. Z. Zhu, and C. T. Chan, Phys. Rev. Lett. 82, 3292 (1999); D. Singh and H. Krakauer, Surf. Sci. 216, 303 (1989). This is also true for W(100).

${ }^{27}$ M. Debe and D. King, Phys. Rev. Lett. 39, 708 (1977).

${ }^{28}$ We have also done calculations for $\{110\}$, in the same manner as we describe here for $\{211\}$. The conclusion is that the $(100)$ is stable with respect to faceting to $\{110\}$ after adsorption of Pd. The major reason is that the angle between $\{110\}$ and (100) is too steep, and thus the area penalty is too high.

${ }^{29}$ C. Herring, Phys. Rev. 82, 87 (1951).

${ }^{30}$ Numerically, $E_{211}$ is about $2 \mathrm{mRy}$ lower than $E_{100}$.

${ }^{31}$ W. K. Lau, M. Phil. thesis, Hong Kong University of Science and Technology (1999).

${ }^{32}$ W. K. Lau, J. G. Che, and C. T. Chan (unpublished).

${ }^{33}$ E. Bauer, Rep. Prog. Phys. 57, 895 (1994).

${ }^{34}$ W. F. Chung and M. S. Altman, Ultramicroscopy 74, 237 (1998).

${ }^{35}$ T. E. Felter, R. A. Barker, and P. J. Estrup, Phys. Rev. Lett. 38, 1138 (1977); see also Refs. 25 and 27.

${ }^{36}$ P. Villars and L. D. Calvert, Pearson's Handbook of Crystallographic Data for Intermetallic Phases (ASM International, Materials Park, 1991) Second Edition, Vol. 4, p. 4432.

${ }^{37}$ Binary Alloy Phase Diagrams, editor-in-chief T. B. Massalski, editors H. Okamoto, P. R. Subramanian, and L. Kacprzak (ASM International, Materials Park, 1990) Second Edition, Vol. 3, p. 2648. 